\title{
Tunability of Liquid Dielectric Resonator Antennas
}

\author{
Steven G. O'Keefe, Member, IEEE, and Simon P. Kingsley, Member, IEEE
}

\begin{abstract}
Dielectric resonator antennas (DRAs) have in the past been used most often at frequencies in the GHz portion of the spectra. This letter presents a novel 50-MHz DRA that uses water as the dielectric. For purposes of keeping the antenna tuned to a specific frequency, and for using the antenna at different frequencies, this antenna can utilize a pump to alter the level of water in the DRA, thus, altering its resonant frequency. An electronic technique for retuning the feed probe is also presented where varactor diodes are biased to provide a good impedance match across a range of approximately 50 to $100 \mathrm{MHz}$. These antennas may provide a compact solution at lower frequencies especially where a directional pattern may be required. The liquid dielectric not only provides frequency tuning but simple deployment and a reducible radar cross section.
\end{abstract}

Index Terms-Dielectric antennas, liquid dielectrics, varactor tuners.

\section{INTRODUCTION}

INCE the first systematic study of dielectric resonator antennas (DRAs) in 1983 [1], interest has grown in their radiation patterns because of their high radiation efficiency, good match to most commonly used transmission lines and small physical size [2]. Most of the configurations reported have used a slab of dielectric material mounted on a ground plane excited by either an aperture feed, in the ground plane, or by a probe inserted into the dielectric material. In some instances a number of feeding probes may be incorporated into a single resonator to achieve the ability to steer the radiation pattern or create a monopulse system [3]. DRAs have most commonly been made from low-loss solid dielectric materials. In particular, ceramics have been used extensively due to their high permittivities and good loss characteristics at high frequencies. There have been reported instances of DRAs being made from liquids which exhibit a relatively high permittivity and low loss [4]. Fig. 1 shows the permittivity and loss tangent of pure water at different temperatures versus frequency [5]. Some investigations have also been performed on saline antennas used as monopole structures [6] and as DRAs [7].

Liquid DRAs open up a new avenue for antenna design where the antenna may have its physical shape altered dynamically. Clearly, the simplest case of this is to change the volume of the resonator in order to change the frequency of resonance. This may be to keep the antenna tuned closely to the frequency of transmission or to use the antenna in another band. A simple

Manuscript received December 20, 2006; revised August 29, 2007.

S. G. O'Keefe is with the Centre for Wireless Monitoring and Applications, Griffith School of Engineering, Griffith University, Nathan, QLD 4111, Australia (e-mail: s.okeefe@griffith.edu.au).

S. P. Kingsley is with Antenova Ltd., Cambridgeshire CB25 9AR, U.K. (e-mail: simon.kingsley@antenova.com).

Color versions of one or more of the figures in this letter are available online at http://ieeexplore.ieee.org.

Digital Object Identifier 10.1109/LAWP.2007.907916

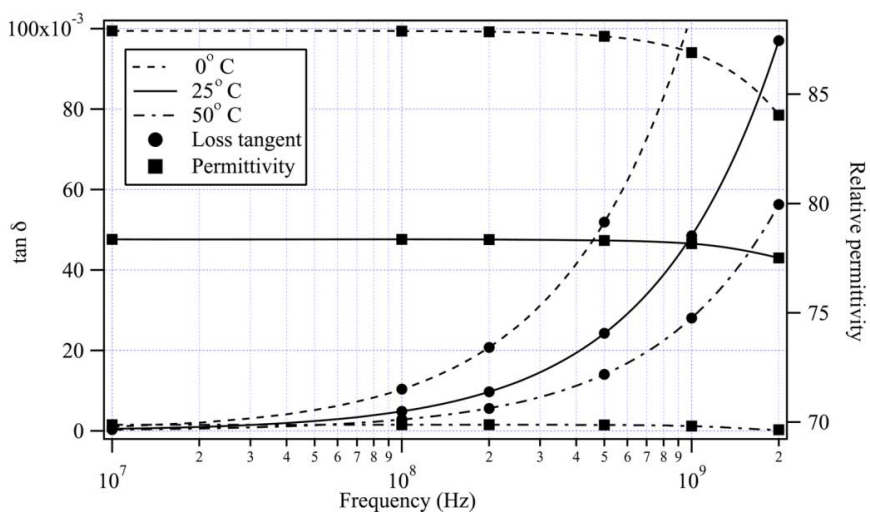

Fig. 1. Permittivity and loss tangent for water at different temperatures versus frequency [5].

pumping arrangement can, therefore, perform the tuning of the antenna. This may also be used to compensate for temperature changes altering the liquids permittivity. This letter presents an experimental investigation of a cylindrical, probe fed, waterfilled DRA, which has its level altered to change the frequency of resonance.

Another advantage of liquid DRAs is the area of prototyping. Often for experimental purposes, DRAs of unusual shape may be required. Simple moulds can provide any shaped DRA when filled with an appropriate liquid. This can also be done with powdered dielectrics [8] although obtaining consistent permittivity can be a problem, especially in unusual shapes. Liquid dielectrics also allow easy access to probes and other structures that may wish to be placed in the dielectric, which is a difficult and often expensive task for solid dielectrics. Computer simulation of course is used to assist in many of these prototyping tasks, but high dielectric simulations may take considerable time. Liquid dielectrics may therefore speed the prototyping procedure in many instances. Small air gaps between the dielectric and the groundplane or between the probe and the dielectric can result in large discrepancies between simulated and measured results [9]-[11]. These air gaps can be difficult to avoid with ceramic dielectric materials but are eliminated when liquid dielectrics are used. Furthermore, liquid dielectrics permit quite complex probe shapes to be experimented with that are not practical to implement inside solid dielectrics.

As the frequency of a DRA is altered, the coupling between the probe and the DRA will alter and hence the resonator will not be correctly coupled giving a poor return loss at the input. To counter this, in a DRA that is being tuned over a wide range of frequencies, the probe must be somehow retuned. A simple electronic method to achieve this is to utilize a varactor diode to retune the probe impedance under the control of an applied dc potential. This is demonstrated experimentally. 


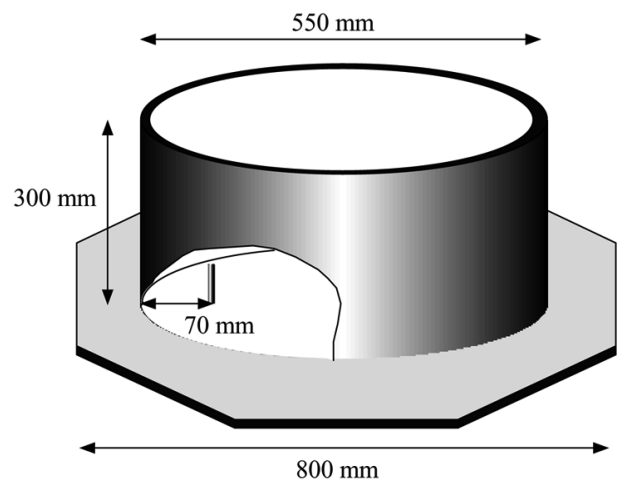

Fig. 2. The structure to contain the water to form the DRA.

\section{THE EXPERIMENTS}

The resonant frequency of the $\mathrm{HEM}_{11 \delta}$ mode in a cylindrical half space DRA can be calculated using (1) taken from [2]. Certain aspect ratios of height (d) and radius (a) give better results and different separation of the resonant modes. Generally a dielectric height of slightly less than the dielectric radius is optimal. The $\mathrm{HEM}_{11 \delta}$ mode is of interest as it has a broadside radiation pattern giving a typical figure 8 dipole pattern in azimuth. This gives two lobes aligned with the axis through the probe and center of the DRA. In many applications, such as direction finding, the nulls are of more importance. This DRA mode has two deep nulls.

$$
f_{o}=\frac{18.972 \times 10^{8}}{2 \pi a \sqrt{\varepsilon_{r}+2}}\left[0.27+0.36\left(\frac{a}{2 d}\right) \pm 0.02\left(\frac{a}{2 d}\right)^{2}\right]
$$

where $d$ is dielectric height and a is dielectric radius.

The cylindrical DRA shown in Fig. 2 is $550 \mathrm{~mm}$ in diameter and is fitted with a single probe $70 \mathrm{~mm}$ from the outer edge. The container holding the water was constructed from plastic of $5 \mathrm{~mm}$ thickness and the octagonal base was constructed of aluminium of $10 \mathrm{~mm}$ thickness. The container was filled with tap water with a temperature of $8^{\circ} \mathrm{C}$. The water was relatively pure with a conductivity of $11 \mu \mathrm{Scm}^{-1}$; ordinary tap water often has a conductivity of $50 \mu \mathrm{Scm}^{-1}$ or greater. The relative permittivity of the water used was estimated to be 87 with a loss tangent of approximately $3 \times 10^{-3}$. At low frequencies it can be seen that water is quite a reasonable dielectric material. The water depth was altered, generally in $10 \mathrm{~mm}$ steps, and the resonant frequency of the $\mathrm{HEM}_{11 \delta}$ mode was measured using a HP 8714B network analyzer operated in reflection $\left(\mathrm{S}_{11}\right)$ mode. A series of measurements were then made to observe the performance of the DRA as the level of water was varied, i.e., simulating the DRA being retuned by pumping water in or out. Experiments were also performed to observe the ability to retune the excitation probe to give optimum matching as the DRA was tuned to a new frequency.

\section{RESULTS}

\section{A. Varying Water Depth}

Initially, a fixed probe length of $175 \mathrm{~mm}$ was used and the water depth was $276 \mathrm{~mm}$. The water depth was then reduced in $10 \mathrm{~mm}$ steps to $156 \mathrm{~mm}$, with a final reading at $151 \mathrm{~mm}$. This

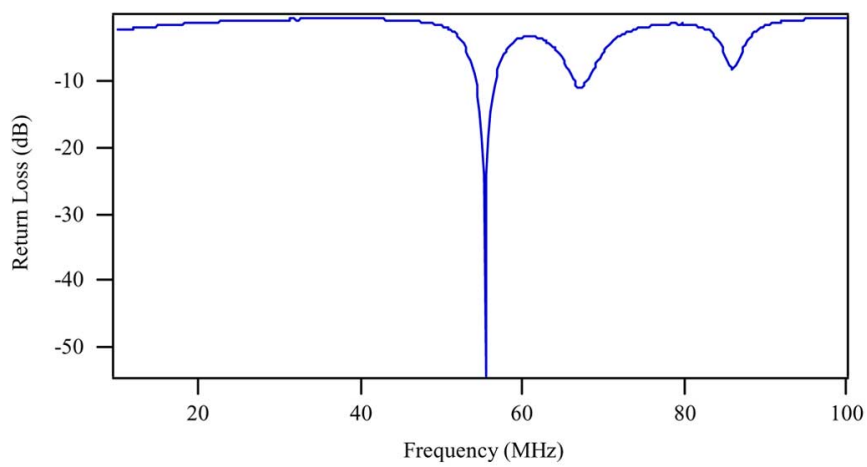

Fig. 3. Return loss of the water filled DRA showing a HEM $\mathrm{H}_{11 \delta}$ mode resonance frequency of $55.5 \mathrm{MHz}$. Water depth $216 \mathrm{~mm}$ and probe length $175 \mathrm{~mm}$.

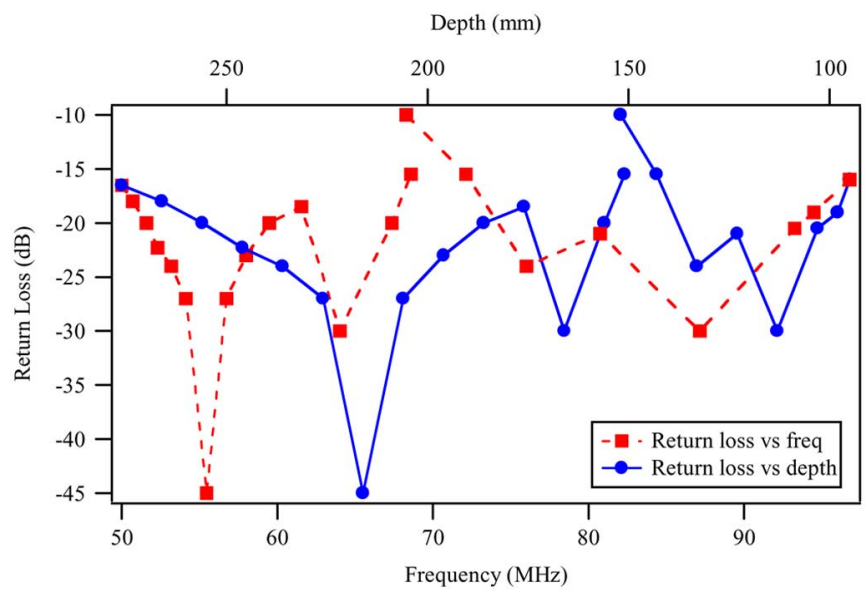

Fig. 4. The variation of return loss with resonant frequency and depth. The right hand section of each line is for the $134 \mathrm{~mm}$ probe and the left hand section for the $175 \mathrm{~mm}$ probe.

change in depth caused a corresponding change in resonant frequency from 50 to $68.6 \mathrm{MHz}$. The return loss at the frequency of resonance was better than $-15 \mathrm{~dB}$ across this frequency range, and was at its' best towards the center of the range. Fig. 3 shows one example of the return loss at a mid-band resonance frequency of $55.5 \mathrm{MHz}$.

At the depth of $151 \mathrm{~mm}$ the return loss had reduced to $-15.5 \mathrm{~dB}$ and so the probe was shortened before proceeding. The remaining readings were taken with a fixed probe length of $134 \mathrm{~mm}$ and the water depth was reduced from $151 \mathrm{~mm}$ in mainly $10 \mathrm{~mm}$ steps to $95 \mathrm{~mm}$. This change in depth caused a corresponding change in resonant frequency from 68.3 to 96.8 MHz. Fig. 4 shows the variation in return loss plotted against water depth and frequency. The discontinuity at the center of each line is where the probe length was changed as described above.

The double-trough nature of the two parts of the plot is caused by an interesting effect; when the aspect ratio (depth/radius) of the antenna is high, around 0.8 , the best return loss is obtained when the top of the probe lies well below the water level. This is the deep trough at the center of Fig. 4 at $55 \mathrm{MHz}$. The second trough, at $64 \mathrm{MHz}$, is caused by the probe breaking the surface as the water level falls. As the aspect ratio is high (0.6) at this stage, this trough does not represent as good a match as the one at $55 \mathrm{MHz}$. However, as the water level continues to go down 


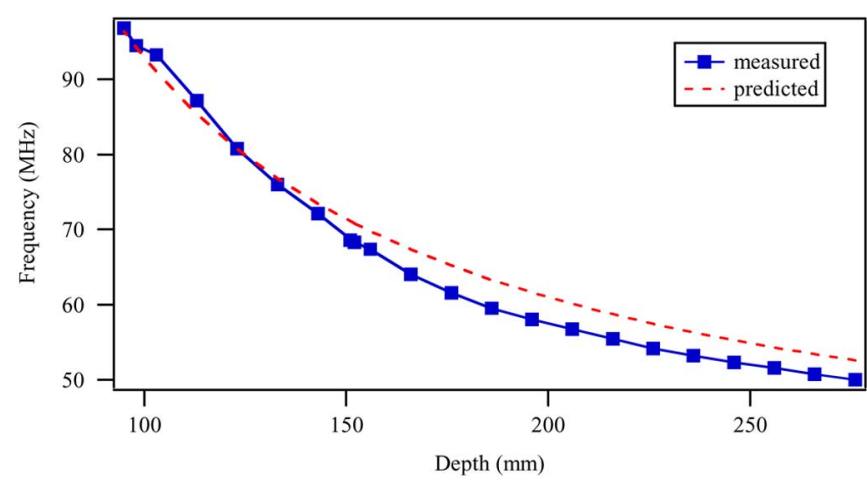

Fig. 5. Predicted and measured resonant frequencies versus water depth.

the antenna develops a low aspect ratio and the next trough at $76 \mathrm{MHz}$, now for the shortened probe well below the surface, is not a particularly good match at $-24 \mathrm{~dB}$ return loss. When the probe does break the surface, a better match of $-30 \mathrm{~dB}$ is obtained at $87 \mathrm{MHz}$. These results demonstrate that the probe should be shorter than the depth of dielectric material for high aspect ratios and comparable with the depth of dielectric material for low aspect ratios, at least for a material with a high dielectric constant.

Fig. 5 shows the variation of resonant frequency plotted against water depth. The resonant frequencies expected from theory are also shown in this figure and the agreement between experiment and theory can be seen to be very good. The water was not temperature controlled so the variation at the higher frequency end is most likely due to the water becoming warmer in the laboratory and, thus, changing its permittivity $\left(\varepsilon_{r}\right)$.

\section{B. Electronic Probe Tuning}

As was seen in the first experiment, the probe that was feeding the DRA needed to be tuned to accommodate the change in frequency as the DRA water level was altered. In that experiment, a manual change of probe length was performed. An electronic tuning method would obviously be much more appropriate.

If the single monopole is replaced by a folded monopole, and the short at the far end replaced by a suitable variable capacitance diode, the probe can be remotely tuned to give a reasonable working match over a fairly wide frequency range $(\sim 8 \%)$. Furthermore, if the DRA height is altered, the probe can be retuned to give the best match over a wide frequency range $(>2: 1$ for the experimental DRA tested).

The same cylindrical DRA was used for this experiment. The single probe was located at $70 \mathrm{~mm}$ from the resonator edge and extended $55 \mathrm{~mm}$ above the groundplane. It was a folded monopole, with $3 \times \mathrm{ZC} 830 \mathrm{~B}$ varicap diodes in parallel at the top, see Fig. 6. Each of these diodes has a nominal 10 to $2 \mathrm{pF}$ tuning range. The probe wire and diodes, which were connected to $\mathrm{N}$-socket inner, were insulated by a layer of varnish, to prevent electrolysis. The tuning voltage was fed into the feed cable via a modified SMA bias-T incorporating a $180 \mathrm{k} \Omega$ series resistor.

Starting at $85 \mathrm{~mm}$, the water depth was increased in steps of 10 to $245 \mathrm{~mm}$ and the probe tuning voltage adjusted to give the best match (deepest null on reflection), as measured on the net-

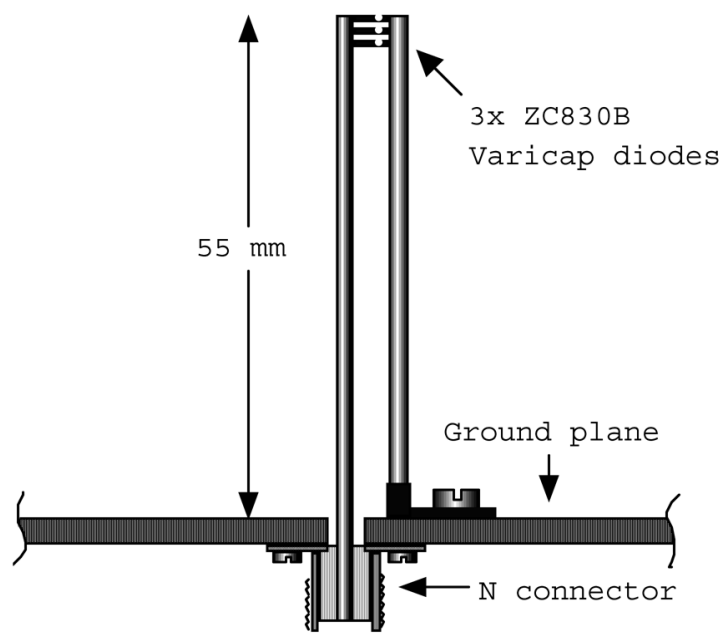

Fig. 6. Tuneable DRA probe.

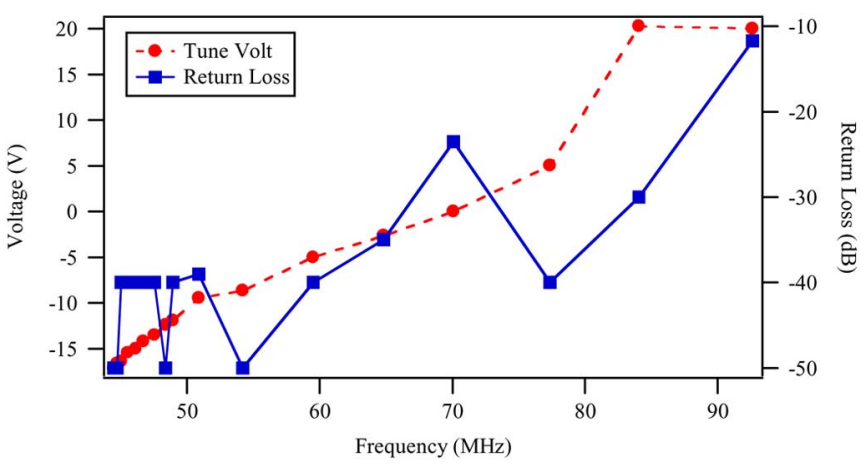

Fig. 7. The best return loss obtained for each frequency as the water level was changed. The tuning voltage required to achieve this match is also shown.

work analyzer. In all cases, this null was very sharp. At $85 \mathrm{~mm}$ water depth, the tuning voltage was at the maximum value for the diodes $(20 \mathrm{~V})$, but the capacitance was still too large for the best match. With some of the probe out of the water, the rate of change in resonant frequency was high and consequently very difficult to tune, with even slight movements of the water having a large effect. Once the probe was covered, tuning was easier, and it was possible to obtain a null of at least $-40 \mathrm{~dB}$. However, in order to get sufficient capacitance with the diodes used, they were required to be forward biased. Fig. 7 shows the return loss achieved over the frequency range and the tuning voltage required to give the best match.

It was also thought useful to examine how far the frequency could be altered by simply tuning the probe and leaving the water level fixed. Using the same configuration, but with a fixed water depth of $165 \mathrm{~mm}$ (covering the probe by $10 \mathrm{~mm}$ ), the frequency was incremented in steps of $0.2 \mathrm{MHz}$ from 46.8 to $51.2 \mathrm{MHz}$ and the probe tuned to give the best match. This frequency range was chosen to give a match better than $-15 \mathrm{~dB}$. Fig. 8 shows the match obtained over the range of frequencies where a match of better than $-15 \mathrm{~dB}$ could be achieved.

\section{DISCUSSION AND CONCLUSION}

Throughout the retunable water DRA experiment, the bandwidth of the antenna, as measured at the $-10 \mathrm{~dB}$ level of the 


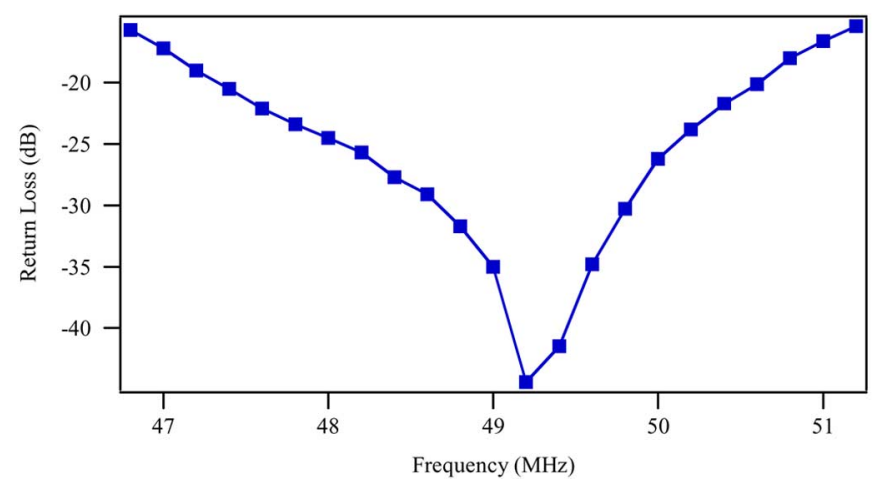

Fig. 8. Return loss achieved by tuning the DRA by adjusting the bias applied to the probe only. Depth of water equals $165 \mathrm{~mm}$ for all frequencies.

return loss, remained at approximately 5\%. Such narrow bandwidths remain a disadvantage of some DRA modes. However, the results presented here show that the antenna can be easily tuned over a bandwidth of $32 \%$ using a single probe of fixed length and $64 \%$ using two probes or a single probe of switchable length. Varying the probe length over a greater range would clearly extend the results. This approach will of course only be suitable for the transmission or reception of narrow band signals, i.e., $5 \%$ of center frequency. The center frequency can, however, be tuned across a band of interest.

The main applications we see at present are for low frequency antennas where space is limited such as on ships or oil rigs. HF and VHF radar, communication and RDF systems could be built with full beamsteering and monopulse processing capabilities in about $11 \%$ of the space occupied by a simple conventional antenna without these capabilities. Liquid antennas also have the advantage of being able to quickly reduce their radar cross section, or lower their center of gravity for transport, by being drained. The problem of liquid motion when the antenna is deployed on a moving platform may be solved by using a support matrix within the vessel such as a solid foam material of the type used to carry fuel within the petrol tanks of racing cars.

Suitable foam materials include low-loss polyurethane "open pore" foams with precisely controlled pore sizes and without cell membranes. These materials have skeletal structures occu- pying about $3 \%$ of the tank volume and are designed to control fuel surges in many types of aircraft and vehicle. The use of gels, rather than low-viscosity liquids, may be another solution to this problem. A plunger style lid over the liquid will also completely maintain the geometry. Obviously water may not be an ideal dielectric in very cold environments due to the large permittivity changes of the solid state. Other liquids cited in [4], heating elements or antifreeze inclusions may be required.

\section{ACKNOWLEDGMENT}

The authors gratefully acknowledge the help of Dr. A. J. T. Whitaker of Sheffield University, U.K., for his help with some of the experiments. U.K. Patent 2370159 and European Patent Application No. 01270927.5 protect some aspects of this work.

\section{REFERENCES}

[1] S. A. Long, M. W. McAllister, and L. C. Shen, "The resonant cylindrical dielectric cavity antenna," IEEE Trans. Antennas Propag., vol. AP-31, pp. 406-412, 1983.

[2] R. K. Mongia and P. Bhartia, "Dielectric resonator antennas-A review and general design relations for resonant frequency and bandwidth," Int. J. Microw. Millimetre-Wave Comput.-Aided Eng., vol. 4, no. 3, pp. 230-247, 1994.

[3] S. P. Kingsley and S. G. O'Keefe, "Beam steering and monopulse processing of probe-fed dielectric resonator antennas," Inst. Elect. Eng. Proc.-Radar, Sonar Navigat., vol. 146, no. 3, pp. 21-125, 1999.

[4] S. G. O'Keefe and S. P. Kingsley, "Radiation efficiency of liquid dielectric resonator antennas," in Nat. Radio Sci. Meet., URSI, Boulder, CO, Jan. 2000, p. 133.

[5] D. R. Lide, CRC Handbook of Chemistry and Physics, 77th ed. Boca Raton, FL: CRC, 1996.

[6] H. Fayad and P. Record, "Broadband liquid antennas," Electron. Lett., vol. 42, no. 3, pp. 133-134, Feb. 2006.

[7] H. Fayad and P. Record, "Wideband saline-water antenna," Wideband Multi-Band Antennas Arrays, pp. 197-201, Sep. 2005.

[8] A. A. Kishk, "Wideband dielectric resonator antenna in a truncated tetrahedron form excited by a coaxial probe," IEEE Trans. Antennas Propag., vol. 51, no. 10, pp. 2907-2912, Oct. 2003.

[9] G. P. Junker, A. A. Kishk, A. W. Glisson, and D. Kajfez, "Effect of an air gap on a cylindrical dielectric resonator antennas operating in the TM 01 mode," Electron. Lett., vol. 30, no. 2, pp. 97-98, 1994.

[10] G. P. Junker, A. A. Kishk, A. W. Glisson, and D. Kajfez, "Effect of an air gap around the coaxial probe exciting a cylindrical dielectric resonator antenna," Electron. Lett., vol. 30, no. 3, pp. 177-178, 1994.

[11] G. P. Junker, A. A. Kishk, A. W. Glisson, and D. Kajfez, "The effect of fabrication imperfections for ground-plane-backed coaxial probe fed dielectric resonator antennas," IEEE Antennas Propag. Mag., vol. 37, no. 1, pp. 40-47, Feb. 1995. 